\title{
MODELO DE SIMULAÇÃO COMPUTACIONAL PARA AVALIAÇÃO DE SEGURANÇA DO TRÁFEGO DE CAMINHÕES EM MINAS A CÉU \\ ABERTO
}

\author{
Welington Monteiro Carneiro
}

welington.carneiro@vale.com

Vale - Diretoria de Ferrosos Sul

Gerência de Planejamento de Longo Prazo Sul

Fazenda Cata Branca, BR-356 - 35450000 - Itabirito MG

\section{RESUMO}

O presente trabalho objetivou desenvolver um modelo de simulação por eventos discretos para estimar a intensidade de tráfego de caminhões fora de estrada em vias de minas a céu aberto e estimar os eventos em que caminhões se encontram com outros em cruzamentos (rotatórias, interseções, bifurcações) com intervalos entre zero (chegada simultânea no ponto) até seis segundos. Para tal, um plano anual futuro de lavra é simulado baseado numa frota pré-dimensionada. Os resultados da análise são usados como uma ferramenta de apoio a ações de segurança de tráfego nas minas, uma vez que permitem trazer previsibilidade do tráfego futuro na mina, permitindo identificar os trechos de maior intensidade de tráfego e os pontos de cruzamentos com maior número de eventos entre zero a seis segundos, que são considerados eventos "críticos", uma vez que podem gerar colisões entre caminhões fora de estrada. Eventos de colisões entre caminhões na empresa estudada não são frequentes, mas caso ocorram, possuem consequências catastróficas e por isso devem ser gerenciados de maneira preventiva no sentido de eliminação e ou mitigação. Os resultados após aplicações em nove minas durante dois anos permitiram mapear como o tráfego nas minas se comporta em função das áreas de lavra, bem como em função das mudanças de lavra ao longo dos trimestres e estão ajudando à empresa a garantir a segurança no tráfego de caminhões fora de estrada nas minas, bem como de veículos de apoio.

Palavras-chave: Simulação, Mina a céu aberto, Análise de Segurança.

\begin{abstract}
This study aimed to develop a discrete event simulation model to estimate the off-roads' intensity of traffic on roads in open pit mines and estimating events in which trucks are at intersections with other ( roundabouts, intersections, junctions ) with intervals between zero (simultaneous arrival on the point ) up to six seconds. For this, an annual future mining plan is simulated based on a pre-sized fleet. The analysis results are used as a tool to support the actions of traffic safety in the mines, since they allow to bring predictability of future traffic in the mine, allowing to identify the roads of more traffic and points of intersections with the highest number of events between zero to six
\end{abstract}


seconds, which events are considered " critical " since it can generate collisions between off-road trucks. Events of collisions between trucks in the studied company are not frequent, but when they occur, have catastrophic consequences and therefore should be managed preventively in order to eliminate or mitigate them. The results after applications in nine mines for two years enabled to map as the mines' traffic behaves according to the mining areas, as well as on the changes in mining over the quarters and are helping the company to ensure safety in traffic off-highway trucks in the mines, as well as support vehicles.

Keywords: Simulation, Open pit mine, Security Analysis.

\section{INTRODUÇÃO}

Considerando o processo de planejamento de lavra num horizonte de médio/curto prazo para um determinado ano futuro relacionado com o tema deste trabalho na empresa onde o mesmo foi aplicado, têm-se as seguintes etapas principais: (i) construção do plano anual de lavra, (ii) dimensionamento de frota e (iii) execução do plano no ano corrente (operação diária). Do plano anual de lavra, sai a informação das rotas a serem utilizadas com as informação de distância e inclinação (grade) das mesmas. Tais informações são as entradas para o processo de dimensionamento de frota, que gera a frota de carga e transporte necessária para realizar o montante de transporte proposto no plano de lavra. No ano corrente, o plano é executado com a frota dimensionada.

Do processo de planejamento descrito, percebeu-se que durante as etapas de construção do plano anual de lavra e dimensionamento de frota, não existia uma análise explícita com relação ao tráfego dos caminhões na mina que um determinado plano e dimensionamento estavam gerando, análise esta com foco na segurança do tráfego operacional nas minas. Aspectos de segurança são observados no processo de planejamento, mas relacionados com aspectos de geométricos das estradas construídas pelos planejadores (largura de via, inclinação, ângulos de curvas, etc).Dessa forma, aspectos negativos como alta concentração de tráfego num determinado trecho da mina, pontos de cruzamento de vias com alto risco, dentre outros, só serão percebidos no ano corrente, o que se considera indesejável no ponto de vista de gerenciamento de aspectos de segurança nas minas.

Uma vez que a empresa entende que segurança é aspecto primordial para suas operações, definiu-se que era necessário avaliar o tráfego operacional nas minas com o objetivo de gerenciar de maneira mais efetiva o aspecto de segurança operacional do tráfego de caminhões nas mesmas, gerando ações preventivas de eliminação ou mitigação dos riscos encontrados. Essa avaliação se deu através da simulação operacional da lavra proposta no plano anual, gerando duas estatísticas principais para o gerenciamento de segurança: intensidade de tráfego de cada trecho da mina e percentual de chegadas críticas (intervalo entre zero a seis segundos) em pontos específicos das estradas, denominados genericamente neste trabalho de cruzamentos.

Este trabalho objetiva apresentar o modelo de simulação desenvolvido e alguns resultados de aplicações do trabalho durante dois anos nas minas da empresa localizadas no estado de Minas Gerais.

\section{LAVRA EM MINA A CÉU ABERTO}

A figura um apresenta um diagrama esquemático do ciclo básico de transporte em uma mina a céu aberto, através dos processos de carregamento, deslocamento 
carregado, descarga e deslocamento vazio. O ciclo se inicia no processo de carregamento, sendo que, após o caminhão ser carregado em uma dada frente de lavra o mesmo é direcionado para um determinado destino (ponto de descarga), podendo ser um britador, uma pilha de homogeneização ou um estoque intermediário. $\mathrm{O}$ mesmo trafega carregado pelas estradas da mina, descarrega no ponto determinado e é novamente direcionado para uma determinada carregadeira que se encontra num dada frente de lavra, trafega vazio pelas estradas da mina e chega novamente ao ponto de carga, apto a iniciar um novo ciclo assim que a carregadeira estiver disponível para o carregamento.

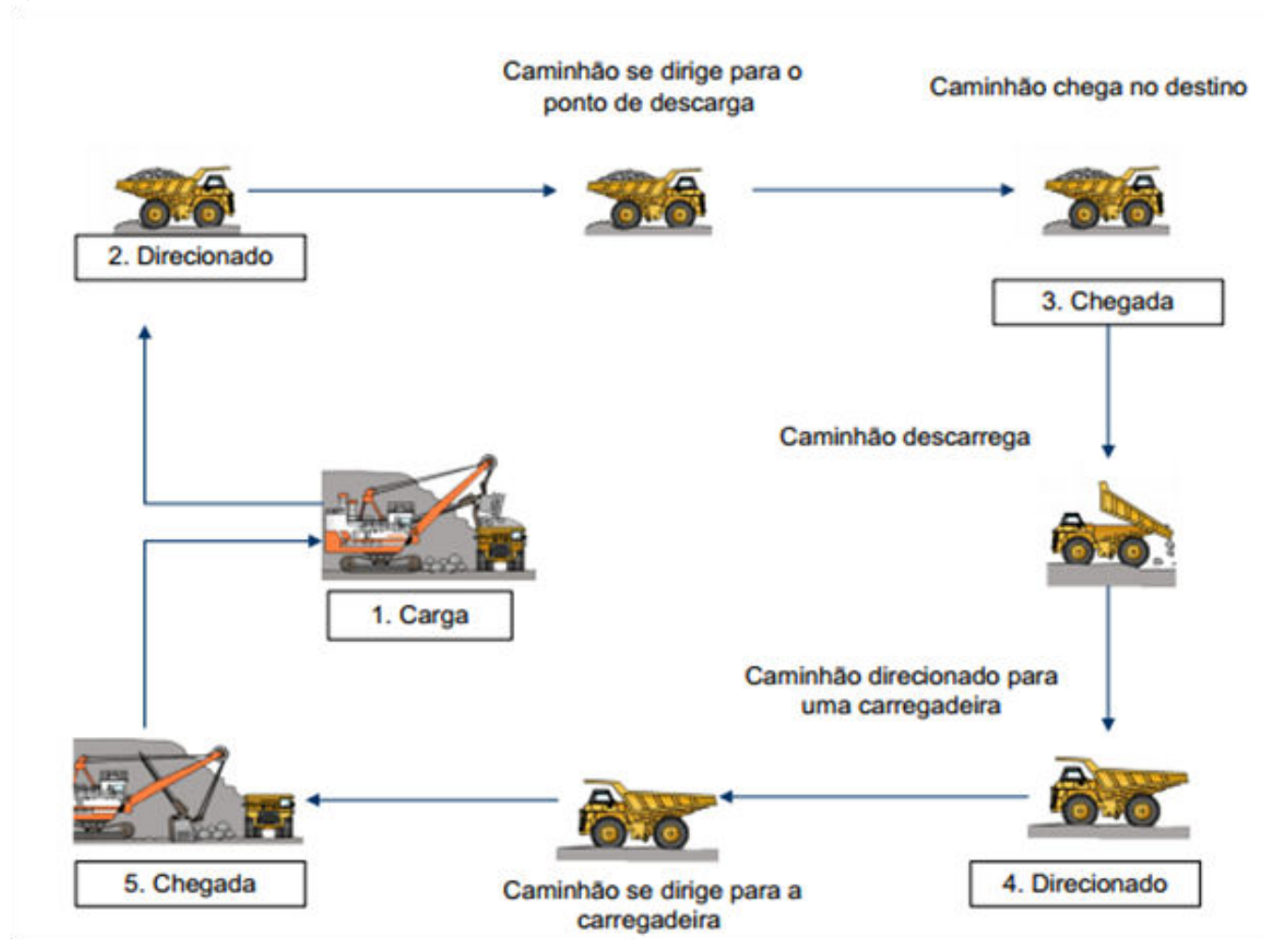

Figura 1: Diagrama do Processo de Carregamento e Transporte. QUEVEDO (2009).

Ressalta-se que o foco deste trabalho está em detalhar os processos de tráfego dos caminhões carregados e vazios entre as origens e destinos da mina, detalhe este necessário para a obtenção das respostas do trabalho que estão altamente ligadas à dinâmica do tráfego dos caminhões na mina. $\mathrm{O}$ autor não encontrou na pesquisa bibliográfica algum trabalho que apresente um modelo de simulação de mina desenvolvido e aplicado especificamente com o objetivo de análise de segurança. A título de revisão de literatura sobre o tema, recomenda-se o trabalho de Quevedo (2009) que apresenta uma extensa revisão bibliográfica da aplicação de simulação nas indústrias minerais. Como referências de modelos de simulação aplicados à minas a céu aberto pode-se citar: Fioroni et al. (2008), Aldrin (2013) e Neto (2006). Com relação a aplicações na área de planejamento de mina cita-se Newman et al. (2010) que fornece uma boa revisão deste tipo de aplicação.

\section{MODELO DE SIMULAÇÃO}

\subsection{ASPECTOS GERAIS}

O Modelo de simulação por eventos discretos foi implementado no software Arena ${ }^{\circledR}$, versão 14.0. A estratégia utilizada neste modelo foi um pouco diferente da 
estratégia utilizada na maioria dos modelos de simulação em minas a céu aberto. Normalmente, os modelos utilizam como variável aleatória de entrada os tempos de deslocamentos entre as origens e os destinos da mina, representados comumente por distribuições de probabilidade. Neste trabalho, as variáveis de entrada são as distribuições de velocidades dos caminhões (cheios e vazios) e as distâncias a serem percorridas pelos mesmos, obtendo assim o tempo de deslocamento, que deixa de ser uma variável de entrada direta. Quevedo (2009) utilizou esta estratégia em seu trabalho, porém utilizando uma distribuição de velocidade para todo um trecho entre origem e destino, independente do perfil da estrada deste trecho.

A estratégia utilizada neste trabalho também se diferencia da utilizada por Quevedo (2009), uma vez que se utiliza distribuição de velocidade por perfil de transporte, o que detalha ainda mais a dinâmica de movimentação dos caminhões. As variáveis de entrada são: (i) distância de um determinado trecho entre uma origem e destino (variável determinística) e (ii) distribuição de probabilidade da velocidade do caminhão por tipo de perfil de transporte (variável aleatória). O perfil de transporte foi dividido em três tipos, baseado no grau de inclinação (\%) da estrada, a saber: (i) Descida: com inclinação menor ou igual a $-3 \%$, (ii) Plano: com inclinação entre $-3 \%$ e $3 \%$ e (iii) Subida: com inclinação maior ou igual a $3 \%$.

Desta forma, um determinado trecho $(\mathrm{Km})$ entre uma origem destino da mina será composto por um ou mais perfis de transporte, conforme descrito acima. Para cada tipo, têm-se um desempenho diferente do caminhão, em termos de velocidade e consequentemente de tempo. Quando a informação de entrada do modelo de simulação é o tempo entre a origem e o destino, tem-se uma informação agregada do tempo necessário para percorrer um determinado trecho, o que será executado pelo modelo de simulação como um evento discreto, definido dentro da estratégia de eventos discretos do software adotado. A estratégia utilizada neste trabalho pode ser considerada um refinamento das informações de deslocamento dos caminhões.

Uma vez que objetivo do modelo proposto é reproduzir com maior fidelidade possível a dinâmica de movimentação dos caminhões nos diversos trechos de uma mina, tal estratégia de utilização de distribuições de velocidade por perfil de transporte se justifica uma vez que uma das variáveis de resposta do modelo (chegadas com intervalos entre 0 a 6 segundos num dado ponto) depende diretamente deste grau de refinamento da informação da velocidade de deslocamento dos caminhões, o que reproduz com mais fidelidade a aleatoriedade inerente ao processo de deslocamento, principalmente pelo fato de que é comum a alternância de perfil de transporte nos acessos de minas a céu aberto (conforme figura 2), por se realizar a lavra em áreas com altitudes diferentes, devido à forma típica de cavas a céu aberto. Desta forma, definiu-se utilizar como variável aleatória de entrada a distribuição de probabilidade das velocidades dos caminhões por tipo de perfil de transporte, baseado no grau de inclinação do mesmo. A figura 1 ilustra a divisão do perfil de transporte:

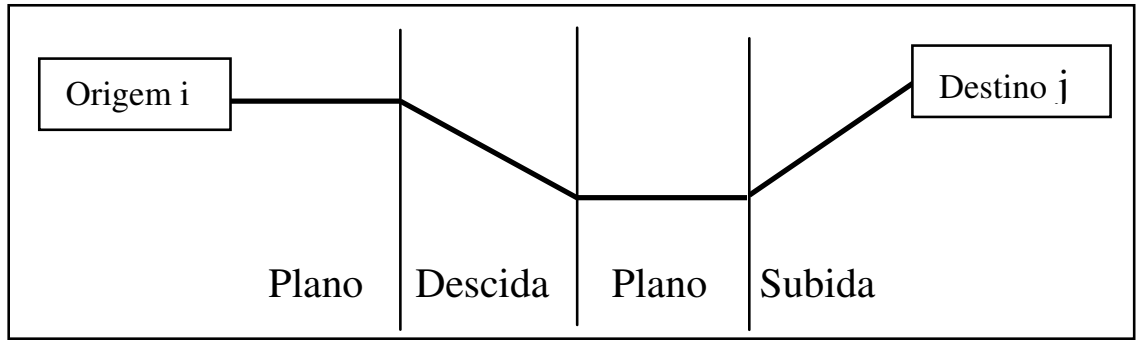

Figura 2: Divisão do perfil de transporte

Cada tipo de perfil possui um atributo numérico, a saber: descida (1), plano (2) 
e subida (3) de maneira que um determinado perfil de transporte passa a ser representado por um vetor, indicando a sequência de tipos de perfis de transporte. Como exemplo, o seguinte vetor: $\mathrm{V}=\{1,2,3,2,3\}^{\mathrm{T}}$ indica que este perfil de transporte entre uma origem e um destino possui a primeira parte em descida, seguido de uma parte plana, uma subida, outra parte plana e outra subida.Complementarmente, o vetor também possui a informação da distância do trecho de cada tipo, formando uma matriz com o número de linhas igual ao número de tipos do perfil e transporte e duas colunas.

Para cada tipo de perfil de transporte foi definido uma distribuição de probabilidade da velocidade (vazio e cheio) de cada tipo de frota de caminhão (pequeno, médio e grande porte), formando assim uma matriz no seguinte formato:

\begin{tabular}{|c|c|c|c|c|c|c|}
\hline & \multicolumn{2}{|c|}{ Pequeno Porte } & \multicolumn{2}{c|}{ Médio Porte } & \multicolumn{2}{c|}{ Grande Porte } \\
\hline Perfil & Cheio & Vazio & Cheio & Vazio & Cheio & Vazio \\
\hline 1 & $\operatorname{Norm}(\bar{x}, \mathrm{~s})$ & $\operatorname{Norm}(\bar{x}, \mathrm{~s})$ & $\operatorname{Norm}(\bar{x}, \mathrm{~s})$ & $\operatorname{Norm}(\bar{x}, \mathrm{~s})$ & $\operatorname{Norm}(\bar{x}, \mathrm{~s})$ & $\operatorname{Norm}(\bar{x}, \mathrm{~s})$ \\
\hline 2 & $\operatorname{Norm}(\bar{x}, \mathrm{~s})$ & $\operatorname{Norm}(\bar{x}, \mathrm{~s})$ & $\operatorname{Norm}(\bar{x}, \mathrm{~s})$ & $\operatorname{Norm}(\bar{x}, \mathrm{~s})$ & $\operatorname{Norm}(\bar{x}, \mathrm{~s})$ & $\operatorname{Norm}(\bar{x}, \mathrm{~s})$ \\
\hline 3 & $\operatorname{Norm}(\bar{x}, \mathrm{~s})$ & $\operatorname{Norm}(\bar{x}, \mathrm{~s})$ & $\operatorname{Norm}(\bar{x}, \mathrm{~s})$ & $\operatorname{Norm}(\bar{x}, \mathrm{~s})$ & $\operatorname{Norm}(\bar{x}, \mathrm{~s})$ & $\operatorname{Norm}(\bar{x}, \mathrm{~s})$ \\
\hline
\end{tabular}

Tabela 1: Matriz de Distribuições de Velocidades por perfil de transporte e porte.

Dessa forma, baseado no atributo do perfil de transporte (1,2 ou 3) e no porte do caminhão (outro atributo), o modelo de simulação acessa a distribuição de probabilidade específica e pela divisão dessa velocidade pela distância do trecho, gera-se o tempo que o caminhão despenderá transitando naquele determinado trecho. Ilustrou-se a distribuição de velocidade na tabela um como sendo todas normais, tendo a média amostral e o desvio-padrão amostral como parâmetro a título de exemplo. As distribuições podem ser determinadas através de um teste de aderência e podem assumir qualquer tipo válido na literatura estatística. Ressalta-se que as distribuições são parâmetros do modelo facilmente ajustáveis/alteráveis.

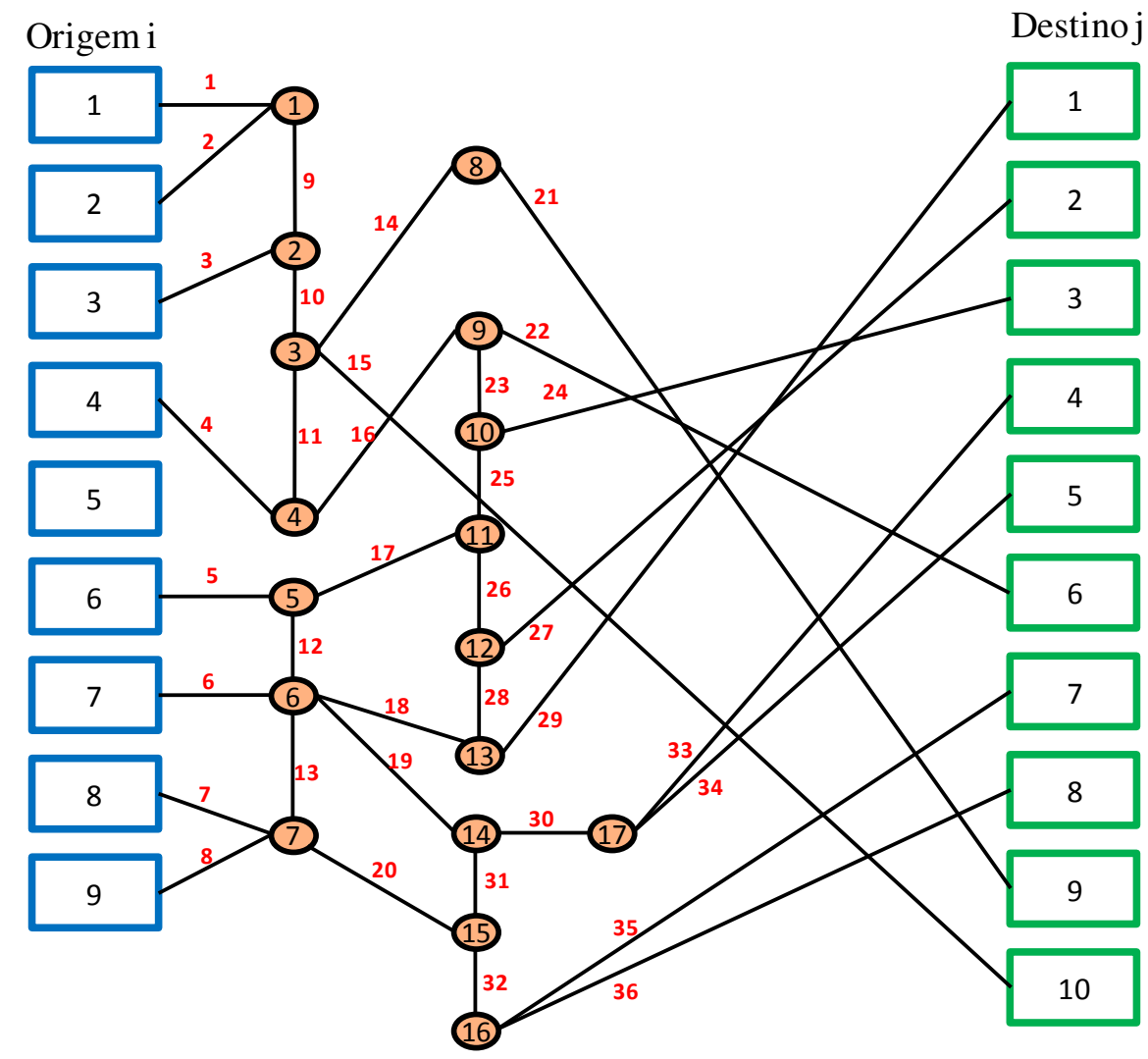

Figura 3: Modelo Simbólico da rede de transporte de uma mina abordada no estudo. 
Neste trabalho, a mina foi modelada como sendo um grafo, em que os vértices (ou nós) representam as origens (frente de lavra), os destinos (britadores e pilhas de estéril) e os pontos mapeados como cruzamento (entroncamentos de vias, bifurcações e rotatórias) e as arestas representam as estradas da mina entre dois vértices. A figura 3 apresenta um exemplo do modelo simbólico utilizado para modelar a rede de transporte das minas. A mina representada na figura dois possui nove origens (frentes de lavra), dez destinos (seis de minério e quatro de estéril), dezessete pontos mapeados como "cruzamentos", trinta e seis partes de estrada denominadas neste trabalho como "trechos". O esquema de divisão do perfil de transporte acontece em cada trecho, em que os mesmo são discretizados conforme o perfil: plano, subida e descida, sendo as partes discretizadas dos trechos denominadas "segmentos".

O exemplo ilustrado na figura três é de uma mina considerada como a maior dentre as nove minas em que o trabalho foi aplicado. A título de dimensão, a menor mina estudada possuía quatro cruzamentos, quatro origens, dois destinos e doze trechos.

\subsection{Modelagem dos Cruzamentos}

Uma vez que o modelo simbólico da mina está construído, a implementação no software de simulação foi feita de maneira que as entidades (caminhões) sejam direcionadas ao longo da rede de transporte, necessariamente tendo que executar o trânsito entre trechos e cruzamentos para se chegar a um destino e executar o sentido contrário para retornar a origem. Conforme exposto, cada trecho é executado com as entidades acessando a informação de velocidade por perfil de transporte do trecho e a informação da distância do mesmo, gerando assim um determinado tempo.

Uma vez que uma das principais variáveis de resposta do modelo é a estatística de chegadas com intervalo entre zero a seis segundos nos pontos de cruzamento, a parte da modelagem que necessitou de maior atenção e desenvolvimento foi a lógica de controle dos caminhões nestes pontos da mina. Para tal, considerou-se que cada cruzamento possui como atributo um conjunto de trechos. Como exemplo representado na figura dois, o cruzamento um possui três trechos ligados a ele, a saber: 1,2 e 9. Dessa forma, criou-se um vetor de trechos por cruzamento que informa quais trechos estão ligados a cada cruzamento. A título de informação, nas nove minas em que o trabalho foi aplicado o máximo de trechos ligados a um mesmo cruzamento foi igual a quatro.

Os pontos modelados como cruzamentos neste trabalho podem ser uma rotatória ou entroncamento (junção) de vias. Ressalta-se que na literatura de transportes, os termos "cruzamento" e "entroncamentos" possuem significados diferentes, mas adotou-se a nomenclatura genérica de cruzamento representando cruzamentos e entroncamentos, assim como na referência [3]. Dessa forma, o modelo de simulação deve ser capaz de reproduzir as lógicas de tráfego dos caminhões em cada tipo de cruzamento considerado, reproduzindo as regras operacionais de preferência de trânsito em situações em que no mínimo dois caminhões se encontram num mesmo ponto de cruzamento. As rotatórias já possuem uma regra de tráfego bem definida, que é a preferência para o caminhão que estiver contornando a mesma. Para tal, modelou-se os pontos de rotatória como "recursos" de capacidade unitária e dessa forma, uma vez que esse recurso estiver ocupado, outro caminhão só poderá acessar o mesmo após a liberação deste recurso. Para os entroncamentos foi necessário conhecer a regra operacional de preferência de cada cruzamento.

A verificação da lógica de controle de tráfego nos cruzamento é realizada num momento discreto do relógio da simulação, momento este determinado pela ocorrência dos eventos em que algum caminhão necessita passar pelo cruzamento e para tal, necessita verificar se o mesmo está liberado. A estratégia de modelagem genérica da 
lógica de controle para todos os cruzamentos se deu através da criação de um conjunto de filas no encontro entre cada trecho e cada cruzamento. Conforme ilustra a figura 3 , no encontro de cada trecho com cada cruzamento existe uma fila, de maneira que ale dos trechos, cada cruzamento também é caracterizado por um conjunto de filas.

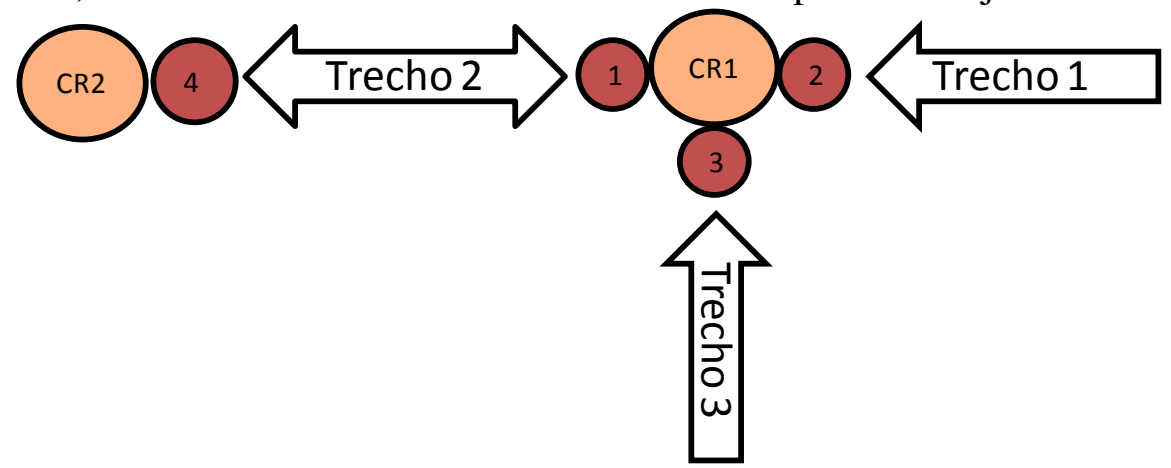

Figura 4: Modelagem dos Pontos de Cruzamento

Considerando o exemplo mostrado na figura 4, após um caminhão percorrer todo trecho um, o mesmo necessariamente deverá ir para a fila número 2 e nesse momento o modelo de simulação percorrerá uma rotina de verificação de liberação de caminhões que informará se esse caminhão poderá prosseguir e passar no cruzamento um ou se o mesmo deverá aguardar a liberação deste cruzamento. Percebe-se que os trechos um e três correspondem a trechos que ligam uma origem a um cruzamento e o trecho dois liga dois cruzamentos, por isso possui uma fila em cada sentido (ida e volta).

Com respeito à prioridade de liberação dos caminhões, além da regra operacional de tráfego nas rotatórias e entroncamentos, outra regra fortemente utilizada é baseada no porte do caminhão e se o mesmo está carregado ou vazio. Conforme a tabela 2, a prioridade máxima é dos caminhões de grande porte cheio, decrescendo até a prioridade mínima, que é dos caminhões de pequeno porte vazio.

\begin{tabular}{cl}
\hline Atributo & \multicolumn{1}{c}{ Significado } \\
\hline 1 & Caminhão Pequeno Porte Vazio \\
2 & Caminhão Médio Porte Vazio \\
3 & Caminhão Grande Porte Vazio \\
4 & Caminhão Pequeno Porte Cheio \\
5 & Caminhão Médio Porte Cheio \\
6 & Caminhão Grande Porte Cheio \\
\hline
\end{tabular}

Tabela 2: Atributos de Prioridade Conforme Porte dos Caminhões.

A rotina de liberação foi implementada em VBA, recurso de programação disponível no software Arena. Tal rotina foi desenvolvida com o objetivo de servir como o procedimento genérico e automático que gerencie a liberação dos caminhões em todos os cruzamentos de uma mina. A figura 5 mostra o fluxograma com o processo decisório empregado. 


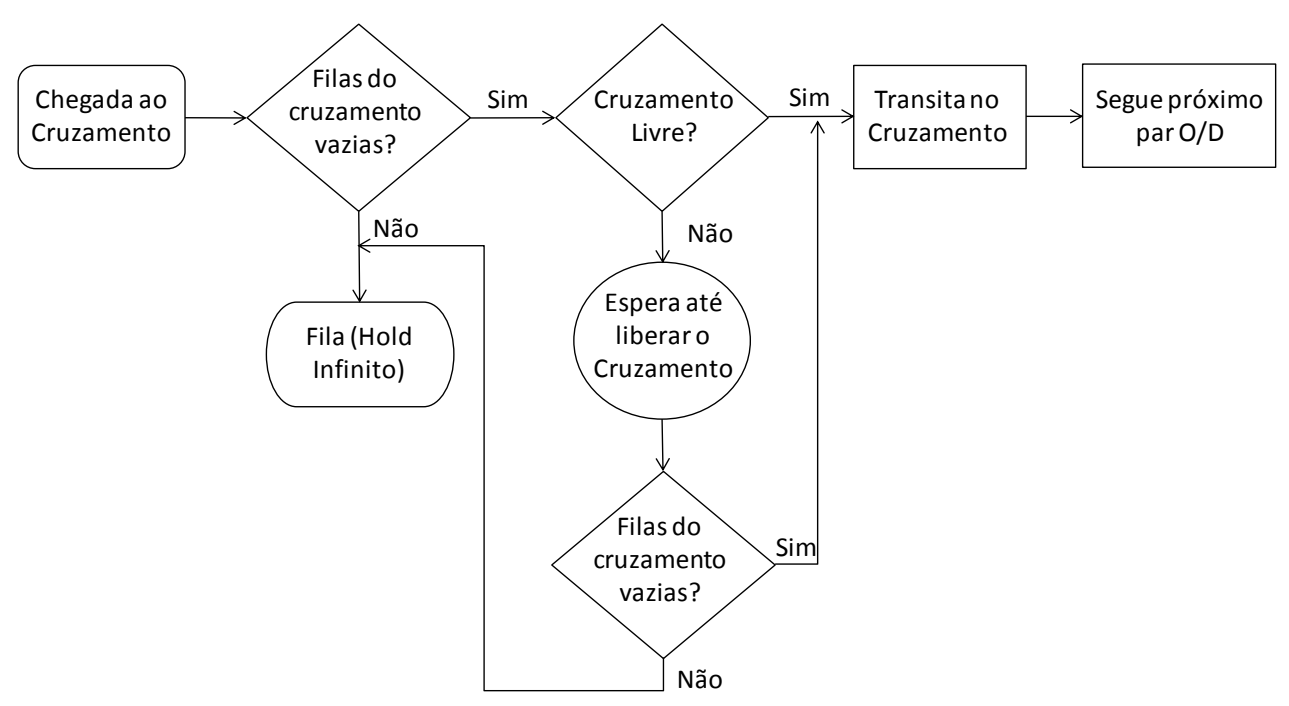

Figura 5: Processo Decisório da Rotina de Controle de Tráfego nos Cruzamentos.

A rotina de liberação foi implementada em VBA, recurso de programação disponível no software Arena. Tal rotina foi desenvolvida com o objetivo de servir de procedimento único para todos os cruzamentos. Conforme o mesmo, após um caminhão chegar a um cruzamento, o mesmo é alocado dentro do software na respectiva fila, conforme figura 3. Neste momento, o relógio da simulação está parado em um tempo discreto e inicia-se o processo de verificação. Para tal processo, quando um caminhão chega a uma fila e necessita esperar o procedimento de liberação, existem dois eventos possíveis de acontecer e as decisões conforme cada um: (i) Demais filas pertencentes ao cruzamento estão vazias e cruzamento vazio, nesse caso o caminhão é liberado para transitar no cruzamento, (ii) Alguma outra fila do cruzamento está ocupada (há outro (s) caminhão (ões) esperando o processo de liberação), nesse caso a rotina verifica as regras de prioridade e libera os caminhões sequencialmente conforme tal regra.

\section{APLICAÇÕES E RESULTADOS}

O modelo de simulação apresentado está sendo utilizado em uma das diretorias de ferrosos da empresa em avaliações de segurança de planos de lavra, focado principalmente em avaliar planos anuais de médio prazo (no mínimo um ano a frente do ano corrente) com o objetivo de fornecer previsibilidade de como se comportará o tráfego nas minas com relação à movimentação de ROM (run of mine) e estéril de um determinado ano. Tal análise tem servido como a primeira etapa no processo de gerenciamento de segurança relacionado com tráfego de caminhões nas minas, sendo definidos a partir do mesmo, planos de ações no sentido de eliminar ou mitigar os riscos mapeados através dos resultados do trabalho. O modelo já foi utilizado em dois anos seguidos de planejamento, sendo aplicado a nove minas em cada ano. Segue alguns exemplos de análises que podem ser feitas utilizando o modelo.

\subsection{RESUltados do MODElO}

O modelo de simulação apresentado possui dois resultados principais, a saber: (i) estatística das chegadas de caminhões nos cruzamentos com intervalo entre zero a seis segundos, consideradas neste trabalho como "eventos críticos" e (ii) estimativa e classificação da intensidade de tráfego dos trechos em leve, moderada e alta.

Toda passagem de um caminhão pelos pontos mapeados como cruzamentos é gravada num arquivo de "log" pelo modelo. Após o tempo de simulação (anual ou 
trimestral) ser alcançado, analisa-se o arquivo de "log" e se extrai do mesmo, para cada cruzamento, todos os casos em que o intervalo entre duas passagens de caminhões seja menor ou igual a seis segundos. Observa-se que as chegadas simultâneas (intervalo de zero segundo) também estão contempladas nesta estatística.

O motivo das chegadas consideradas como "críticas" serem no intervalo entre zero a seis segundos advém do fato que, conforme a tabela 3, o tempo mínimo necessário para percepção, reação e frenagem de caminhões com porte para transportar mais de 180 toneladas é de seis segundos. Uma vez que nas nove minas abordadas se possui todos os tipos de frotas de caminhões (menor que 45 toneladas até maior que 180 toneladas) considerou esse tempo como o limite para as chegadas classificadas como "críticas". Ressalta-se que, esse valor é um parâmetro da rotina que contabiliza tais eventos nos arquivos de "log". Como todas as passagens dos caminhões são gravadas, caso se queira analisar as chegadas até 10 segundos (em vez de chegadas até seis segundos), não é necessário rodar o modelo de simulação novamente, somente contabilizar o novo valor nos arquivos de "log".

\begin{tabular}{|c|c|c|c|}
\hline $\begin{array}{c}\text { Peso do } \\
\text { Caminhão }(\mathbf{t})\end{array}$ & $\begin{array}{c}\text { Tempo de } \\
\text { Percepção }(\mathbf{s})\end{array}$ & $\begin{array}{c}\text { Tempo de } \\
\text { Frenagem (s) }\end{array}$ & Total (s) \\
\hline$<45$ & 1,5 & 0,5 & 2 \\
\hline $45<\mathrm{t}<90$ & 1,5 & 1,5 & 3 \\
\hline $90<\mathrm{t}<180$ & 1,5 & 2,8 & 4,3 \\
\hline$>180$ & 1,5 & 4,5 & 6 \\
\hline
\end{tabular}

Tabela 3: Tempo de reação de frenagem em função do peso do caminhão - Manual de Estradas de Mina Vale - OBS: Considerando um fator de atrito de $\mathrm{f}=0,55$ e velocidade de $40 \mathrm{Km} / \mathrm{h}$.

Outro resultado do trabalho é a estimativa e a classificação da intensidade de tráfego em cada trecho da mina conforme a tabela 4. Ressalta-se que os percentuais considerados nos critérios de classificação da intensidade de tráfego em um trecho como: (i) leve, (ii) moderada e (iii) alta foram definidas por "experiência" práticas das pessoas envolvidas no trabalho e podem ser facilmente alteradas conforme uma melhor adequação. Utilizou-se a mesma regra para todas as nove minas em que o trabalho foi aplicado, mas cada mina poderia ter seu próprio critério, caso tal consideração seja mais representativa da realidade da mina na prática.

\begin{tabular}{cc}
\hline$\%$ de Tráfego do Trecho & Classificação de Intensidade \\
\hline$<6 \%$ & Leve (cor verde) \\
$>6 \%$ e $<13 \%$ & Moderado (cor laranja) \\
$>13 \%$ & Alta (cor vermelha) \\
\hline
\end{tabular}

Tabela 4: Classificação da Intensidade de Tráfego. Fonte: Do Autor.

\subsection{ANÁLISE DO EFEITO DO PORTE DA FROTA DE CAMINHÕES NO TRÁFEGO DAS MINAS}

A escolha do tipo de frota de transporte a ser utilizada numa mina possui vários fatores preponderantes, principalmente custo. O dimensionamento da frota a ser utilizada (porte e quantidade de caminhões) possui como input principal a movimentação necessária na mina (ROM, Estéril, Produto ou Outras Movimentações). Uma pergunta que surgiu no âmbito desse trabalho foi: Qual é o efeito do porte da frota de caminhões nos indicadores de segurança considerados neste trabalho?

Para tal, foi escolhida uma mina e foram configurados dois cenários: cenário (i) composto por 8 caminhões de pequeno porte $(38 \mathrm{t}$ ) e 14 caminhões de médio porte (70 t), totalizando uma capacidade da frota de 1284 toneladas e o cenário(ii) composto por 4 caminhões de médio porte $(70 \mathrm{t})$ e 7 caminhões de grande porte $(146 \mathrm{t})$, totalizando 
uma capacidade de frota de 1302 toneladas. Dessa forma, o cenário dois possui metade do número de caminhões do cenário um com o objetivo de analisar qual seria o efeito da redução de $50 \%$ da frota nos indicadores de segurança considerados neste trabalho.

\begin{tabular}{lccccc}
\hline & PP & MP & GP & $\begin{array}{c}\text { No Total de } \\
\text { Caminhões }\end{array}$ & $\begin{array}{c}\text { Capacidade Frota } \\
\text { (t) }\end{array}$ \\
\hline Cenário 1 & 8 & 14 & 0 & 22 & 1284 \\
Cenário 2 & 0 & 4 & 7 & 11 & 1302 \\
\hline
\end{tabular}

Tabela 4: Composição dos Cenários de teste.

A tabela 6 mostra o resultado do cenário um e dois com relação às chegadas críticas nos cruzamentos. O cenário dois representa a redução de $50 \%$ a frota. Os resultados mostraram que o número de chegadas críticas foi reduzido em $73 \%$. Tal resultado é bem interessante e mostra que o efeito da redução da frota com relação às chegadas críticas foi percentualmente maior que o percentual de redução da frota. Podese explicar o mesmo analisando o fato de que no cenário um havia oito caminhões de pequeno porte e 14 de médio porte, que são caminhões que desenvolvem uma maior velocidade, o que é refletido em caminhões chegando aos pontos de cruzamento com maior intensidade. No cenário dois, além da frota ter sido reduzida pela metade, há somente quatro caminhões de médio porte e sete caminhões de grande porte, que são caminhões que alcançam uma dinâmica menor em termos de desenvolvimento de velocidade comparados com os caminhões de pequeno e médio porte.

\begin{tabular}{ccc}
\hline Cruzamento & \multicolumn{2}{c}{ № Chegadas Críticas } \\
\cline { 2 - 3 } & Cenário 1 & Cenário 2 \\
$\mathbf{1}$ & 33192 & 11235 \\
$\mathbf{2}$ & 72285 & 19869 \\
$\mathbf{3}$ & 803 & 124 \\
$\mathbf{4}$ & 48308 & 10267 \\
\hline
\end{tabular}

Tabela 6: Resultados (chegadas Críticas) - Cenários 1 e 2.

\subsection{ANÁLISE DO TRÁFEGO COM A MUDANÇA DE TRIMESTRE}

A análise a seguir se baseou na seguinte pergunta: Qual é o efeito da mudança de trimestre (consequentemente de um plano de lavra trimestral) nos indicadores de segurança? Tal análise corresponde a uma situação real de uma das minas estudadas, em que se analisou o efeito da mudança de plano de lavra do $1^{\circ}$ para o $2^{\circ}$ trimestre. A figura dez mostra a representação dos trechos dispostos na mina alvo do estudo.

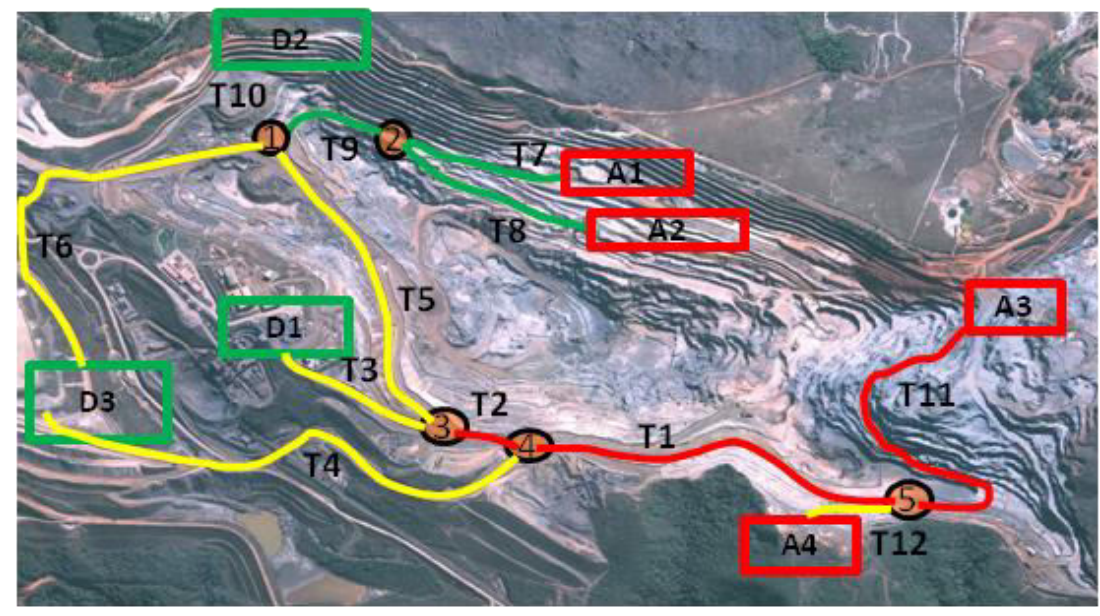

Figura 10: Ilustração dos trechos na mina alvo do estudo $-1^{\circ}$ trimestre. 
A figura onze mostra a distribuição da intensidade de tráfego percentual para o primeiro trimestre e as figuras doze e treze mostram os trechos da mina considerados no segundo trimestre e a intensidade de tráfego percentual para o segundo trimestre.

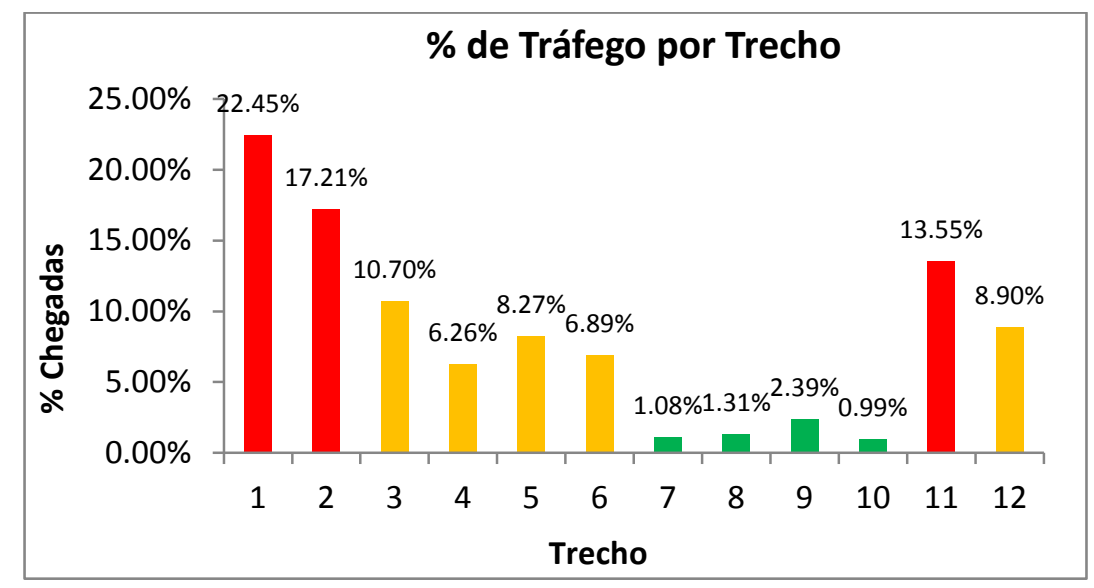

Figura 11: Intensidade de tráfego por trecho $-1^{\circ}$ trimestre.



Figura 12: Ilustração dos trechos na mina alvo do estudo $-2^{\circ}$ trimestre.

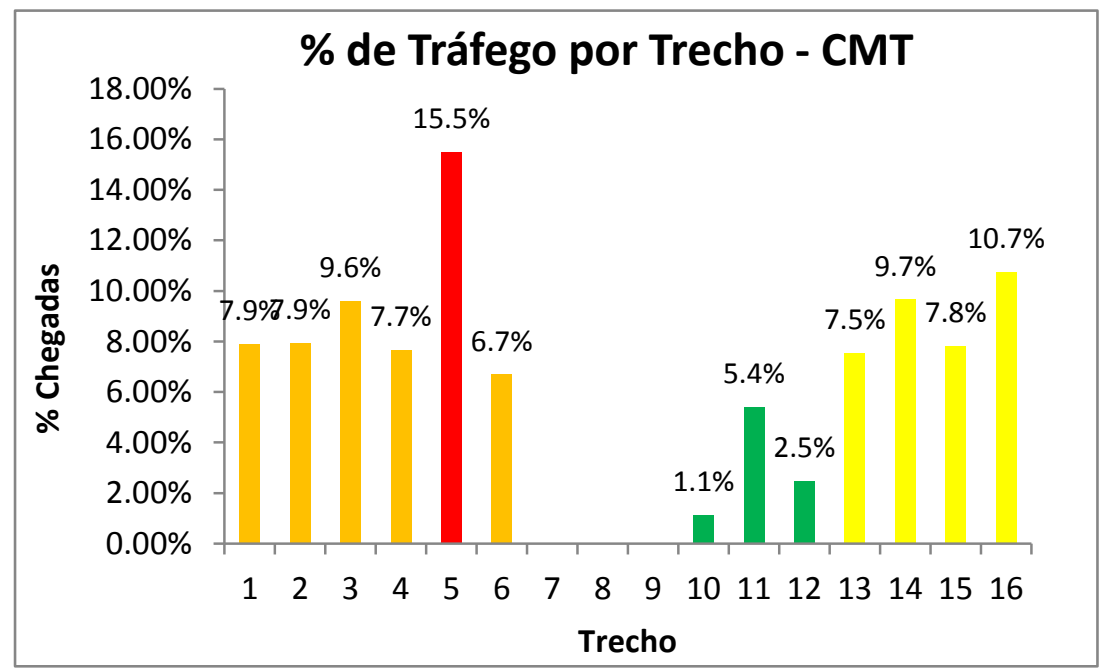

Figura 13: Intensidade de tráfego por trecho $-2^{\circ}$ trimestre. 
Comparando as figuras dez e doze, percebe-se que devido à mudança do primeiro para o segundo trimestre, a região da mina cujos trechos possuem alta intensidade de tráfego também mudou. No primeiro trimestre os trechos um, dois e onze possuíam altas intensidades de tráfego e no segundo trimestre percebe-se que tal situação se deslocou para o trecho cinco, em virtude das diferenças de áreas e massas a ser lavrada. A utilidade desta análise está no fato de que a mesma permite analisar como se comporta o tráfego na mina ao longo dos trimestres, meses e até semanas, o que pode ser uma informação inicial no processo de gerenciamento preventivo de segurança na mina.

\section{CONCLUSÃO}

O desenvolvimento e utilização do modelo simulação a eventos discretos permitiu observar os aspectos relacionados ao tráfego de mina o mais próximo da realidade, detalhando a dinâmica de deslocamento dos caminhões ao longo das estradas da mina. O modelo foi e está sendo aplicado na empresa em questão para ações preventivas com relação à segurança do tráfego nas minas e forneceu informações uteis para ações relacionadas a cruzamentos críticos e trechos com grande intensidade de fluxos. Como contribuição às aplicações da área, o trabalho apresentou uma maneira mais detalhada de geração dos tempos entre as origens e destinos na mina (principal fonte estocástica do sistema) através da utilização de distribuições de probabilidade das velocidades dos caminhões para cada tipo de perfil de transporte das estradas da mina (plano, subida e descida) o que permite detalhar com mais fidelidade à dinâmica aleatória do tráfego dos caminhões, conforme a realidade.

\section{REFERÊNCIAS BIBLIOGRÁFICAS}

[1] QUEVEDO, J.M.G. Modelo de simulação para o sistema de gerenciamento e transporte em mina a céu aberto. Dissertação (Mestrado em Engenharia de Produção) - Pontifícia Universidade Católica do Rio de Janeiro, PUC-RIO, Rio de Janeiro, 2009.

[2] FIORONI, M. M. et al. Concurrent simulation and optimization models for mining planning, Winter Simulation Conference, p. 759-767, 2008.

[3] ALDRIN, G.M. Simulação das operações de lavra da mina de brucutu utilizando um modelo de programação linear para alocar os equipamentos de carga. Dissertação (Mestrado) - Universidade Federal de Ouro Preto. Escola de Minas. Departamento de Engenharia de Minas, Ouro Preto, 2013.

[4] NETO, L.R.C. Modelagem e Simulação da cadeia Produtiva do Minério de Ferro. Dissertação (Mestrado) - Escola Politécnica da Universidade de São Paulo. Departamento de Engenharia de Transportes, São Paulo, 2006.

[5] NEWMAN, A. M. et al. A review of operations research in mine planning, Interfaces, v. 40, n. 3, p. 222-245, 2010.

[6] Manual de Estradas de Mina. Vale. 2011

[7] Sinalização de Cruzamentos e de Entroncamentos. Instituto de InfraEstruturas.2001.Acessadoem:http://www.inir.pt/portal/LinkClick.aspx?fileticket= 2lxDfPmAlXA\%3D\&tabid=116\&mid=487\&language $=$ pt $-\mathrm{PT}$. 